\title{
Soluble MRP8/14 recruits neutrophils via TLR4
}

A new report by Pruenster et al. reveals a novel Toll-like receptor 4 (TLR4)mediated mechanism through which circulating MRP8/14 complex (also known as S100A8-9 complex, or calprotectin) rapidly activates $\beta 2$ integrins and promotes neutrophil slow rolling and adhesion to inflamed vascular endothelium. Although elevated serum levels of soluble MRP8/14 complex are used as a biomarker of inflammation, the exact role of circulating MRP8/14 complex in inflammation had-until now-remained elusive.

Markus Sperandio, the study's corresponding author, describes these exciting findings: "We hypothesized that locally released MRP8/14 acts in a proinflammatory manner and plays a role in activating neutrophils during recruitment. Our study now shows that E-selectin-triggered adhesion critically depends on an MRP8/14-dependent autocrine extracellular activation loop."

In vitro and ex vivo flow chamber assays were used to assess neutrophil adhesion, in which binding of the neutrophil cell surface marker PSGL-1 (P-selectin glycoprotein ligand 1) to E-selectin on vascular endothelial cells led to the release of soluble MRP8/14 complex by neutrophils. Interestingly, although P-selectin also bound to PSGL-1 (albeit to a different epitope), this interaction did not result in MRP8/14 release. MRP8/14 complexes bound to neutrophil-expressed TLR4 in an autocrine (local) manner, triggering an intracellular signalling cascade involving the adapter protein MyD88 and Rap1, a small GTPase that regulates the binding affinity of $\beta 2$ integrins and, thereby, controls neutrophil rolling velocities and adhesion. An additional finding was the rapidity of TLR4-dependent $\beta 2$ integrin activation; other forms of TLR4 signalling are mostly mediated by transcriptional effects. The researchers also used paquinimod, an inhibitor of MRP8/14, to study the effects of circulating MRP8/14 complex, which enabled them to avoid interfering with the intracellular function of MRP14 as occurs in genetically modified mice.

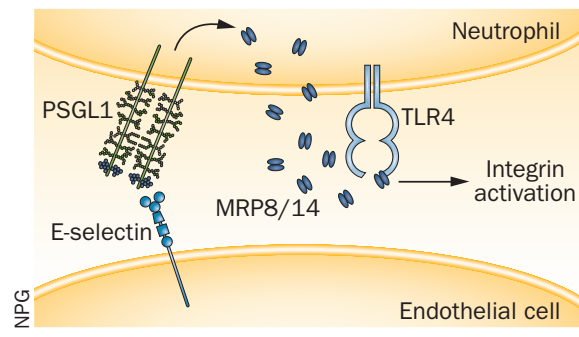

"We would like to further elucidate the intracellular signalling events downstream of E-selectin and PSGL-1 leading to the release of MRP8/14," comments Sperandio. "Additionally, some of us are currently generating blocking monoclonal antibodies against MRP8/14, which might be a potent new therapeutic tool to treat patients with arthritis or other autoimmune diseases."

Caroline Barranco

Original article Pruenster, M. et al. Extracellular MRP8/14 is a regulator of $\beta 2$ integrin-dependent neutrophil slow rolling and adhesion. Nat. Commun. doi:10.1038/ncomms7915 